Saudi Journal of Pathology and Microbiology

Abbreviated Key Title: Saudi J Pathol Microbiol

ISSN 2518-3362 (Print) |ISSN 2518-3370 (Online)

Scholars Middle East Publishers, Dubai, United Arab Emirates

Journal homepage: http://scholarsmepub.com/sjpm/

Original Research Article

\title{
Prognostic Significance of Metastatic Lymph Node Ratio in Gastric Cancer
}

\author{
Dr. Aysha Femy ${ }^{1}$, Dr. Anuradha C.K.Rao ${ }^{2 *}$ \\ ${ }^{1}$ Postgraduate, Department of Pathology, Yenepoya Medical College, Deralakatte, Mangalore \\ ${ }^{2}$ Professor, Department of Pathology, Yenepoya Medical College, Deralakatte, Mangalore
}

DOI: 1 10.36348/sjpm.2019.v04i11.002 $\quad$ | Received: 30.10.2019| Accepted: 06.11.2019| Published: 12.11 .2019

*Corresponding author: Dr. Anuradha C.K Rao, MD. DNB

\section{Abstract}

Objective: The objective of this study was to analyze the prognostic value of lymph node ratio in patients with gastric cancer. Methods: A total of 22 cases of total and subtotal gastrectomy received in the period of January 2015 to July 2018 in the department of pathology of a tertiary care hospital were assessed. The surgical data and the histopathological findings were assessed using definitive criteria. Pearson's correlation coefficient and chi-square test was used to study the relation between the lymph nodes showing metastasis and the total number of lymph nodes harvested. The relation of LNR with the other clinicopathological parameters was studied. Results: A cut off value of 0.25 was taken and the cases were grouped into low grade and high grade LNR group. In this study we found that the LNR was associated with the number of metastatic lymph nodes, irrespective of the total number of lymph nodes dissected. Hence it could be used as a prognostic indicator even when less number of lymph nodes is retrieved. Conclusions: LNR could be considered as a valuable prognostic factor and could be used as an additional tool with the TNM staging system to assess prognosis in patients post-surgery. It is especially useful in cases where prescribed number of lymph nodes cannot be retrieved.

Keywords: Gastric cancer, Lymph node ratio, prognostic factor, TNM stage.

Copyright @ 2019: This is an open-access article distributed under the terms of the Creative Commons Attribution license which permits unrestricted use, distribution, and reproduction in any medium for non-commercial use (NonCommercial, or CC-BY-NC) provided the original author and sources are credited.

\section{Prognostic significance of metastatic lymph node ratio in gastric cancer}

\section{INTRODUCTION}

Gastric cancer is the fifth most common malignancy with $10,33,701$ (5.7\%) new cases being reported in 2018 according to the reports of Globocan and the $3^{\text {rd }}$ leading cause of cancer deaths worldwide[1]. The proposed incidence for India is expected to go up to 50,000 annually in the year 2020[2]. While, surgical resection is the mainstay of treatment for gastric carcinoma, clinicopathological staging is essential for postoperative therapies, with Union for International Cancer Control (UICC)/American Joint Committee on Cancer (AJCC) TNM staging system being the most widely used. It classifies patients into different stages based on the depth of primary tumor invasion ( $\mathrm{T}$ stage), regional lymph node metastases ( $\mathrm{N}$ stage) and distant metastases (M stage)[3].

The 5-year survival rate of patients with gastric carcinoma with lymph node metastasis has been found to be significantly lower than in those without. The AJCC has emphasised much on the lymph node dissection and has stated in the $8^{\text {th }}$ edition that adequate dissection of the regional lymph nodes is essential to ensure appropriate $\mathrm{N}$ staging. It is also recommended that at least 16 regional nodes should be examined; evaluation of more than thirty nodes is been considered desirable [5]. Furthermore, accurate $\mathrm{pN}$ staging is considered crucial for decision making of post-surgical neo-adjuvant therapies, improving the efficacy [6]. While the older case studies have reported that the total number of metastatic lymph nodes has a significant prognostic value in gastric carcinomas, the newer reports have demonstrated that the lymph node ratio is of better prognostic importance [6]. Also many-a-times retrieval of 16 lymph nodes as suggested by AJCC may not be possible. This inadequate and inaccurate examination of lymph nodes leads to a phenomenon known as stage migration. Hence studies have opted to use the lymph node ratio as a better prognostic indicator. LNR is proposed to be a simple and reproducible prognostic factor, even in the cases of limited lymph node dissection, reducing the phenomenon of stage migration [3]. 
Lymph node ratio is defined as a ratio of number of metastatic lymph nodes to the total number of lymph nodes retrieved [6]. The cut-off value of lymph node ratio which could be used to distinguish different prognoses is not clearly defined. LNR $>0.25$ has been predicted to show a worse overall survival according to a few studies [4].

This study investigates if LNR could be used as a reliable indicator of prognosis in patients with gastric carcinoma and to assess its significance in comparison with other prognostic factors.

\section{OBJECTIVES}

To study the clinico-pathological factors affecting the prognosis of gastric cancer and to study the association of these factors with lymph node ratio. Also analyse the prognostic value of lymph node ratio in these patients.

\section{MATERIALS AND METHODS Study design}

A retrospective study was conducted in the Department of pathology of a tertiary care hospital. Twenty-two cases of total and subtotal gastrectomy received in the period of January 2015 to july 2018 were included.

\section{Ethics statement}

This study was performed after approval from the institutional Ethical committee.

\section{Inclusion criteria}

All the total and subtotal gastrectomy specimens for carcinoma stomach received during the course of this study irrespective of the variants or subtype were included.

\section{Exclusion criteria}

All the gastric biopsy specimens and all gastrectomy / partial gastrectomy specimens without any malignancy on histopathology.

Based on the above mentioned criteria, 22 cases of total and subtotal gastrectomy was included. The records were collected and reviewed from the Medical records department. Surgical data and the histopathological findings were assessed. The lymph nodes were thoroughly dissected out from the specimen. These were then stained with $H \& E$ and examined. The clinicopathological data recorded were age, sex, location, tumour size, histopathological type, Borrman type, histological grade, gastritis, hyperplastic areas, intestinal metaplasia and dysplasia in adjacent stomach, depth of tumor invasion, total number of lymph nodes isolated, the number of metastatic nodes, lymph node ratio, lymphovascular invasion, perineural invasion, TNM stage, Type of operation, the resected margins and post gastrectomy radiation / chemotherapy.
The follow-up data of these patients for a minimum of 1 year was collected.

\section{Classification of lymphadenectomy}

Based on the current CAP Protocol, the dissected lymph nodes were grouped as pNX (Regional lymph nodes cannot be assessed); pNO (No regional lymph node metastasis); pN1 (Metastasis in $1 / 2$ regional lymph nodes); pN2 (Metastasis in 3-6 regional lymph nodes); pN3 (Metastasis in 7 or more regional lymph nodes); pN3a (Metastasis in 7 to 15 regional lymph nodes); pN3b (Metastasis in 16 or more regional lymph nodes)[5]. The metastatic lymph node ratio was further calculated as the ratio of pathological metastatic lymph node number and total number of lymph nodes dissected.

Various previous studies have proposed different optimum cut off value of LNR [8]. In this study the cut off value was taken as 0.25 as done by $\mathrm{Hu}$ et al. in their study [4]. Accordingly, patients were grouped into low grade LNR group (LNR <0.25) and high grade LNR group ( LNR>0.25).

\section{STATISTICAL ANALYSIS}

The data were entered into Microsoft excel sheet and analyzed using the software SPSS 23 version. Pearson's correlation coefficient and chi-square test was used to study the relation between the lymph nodes showing metastasis and the total number of lymph nodes submitted. Also the relation of metastatic lymph node and LNR, and LNR and total number of lymph nodes retrieved was assessed. P-value $<0.05$ was considered significant.

\section{RESULTS \\ Clinicopathological characteristics}

A total of 22 patients were included in this study. The clinicopathological parameters of these patients are summarized Table 1. The median age of patients with gastric carcinoma in our study is 59 years (range 30-74years). Majority of the patients were males $(68 \%)$ and $32 \%$ were females. Personal history of these patients revealed that all of them followed a mixed diet and no family history of any cancer was known. Regarding tumor location, antrum and pylorus (77\%) was the most common site of involvement followed by fundus and cardia. Forty six percent of these cases had a tumor size more than $6 \mathrm{~cm}$. As for the histological type of tumor, adenocarcinoma intestinal type was the most common (55\%), 45\% had adenocarcinoma diffusely infiltrating which included 3 cases each of carcinoma with mucinous areas and signet ring cell carcinoma. Also, $77 \%$ of these cases were classified as Bormann type IV and $23 \%$ as Bormann type III. According to the AJCC $8^{\text {th }}$ edition of classification of tumors $6(27 \%)$ cases were categorized as IIA, $6(27 \%)$ as IIB, $4(18 \%)$ as IIIA, $3(14 \%)$ as IIIB and $3(14 \%)$ as IIIC disease. 
Well differentiated carcinoma was seen in $13 \%$ cases, $46 \%$ and $41 \%$ had moderately differentiated and poorly differentiated tumors respectively.

Lymphovascular invasion was noted in $50 \%$ and was found to have significant correlation $(\mathrm{p}<0.05)$ with higher number of metastatic lymph nodes. Perineural invasion was seen in $50 \%$ of the cases.

In this study, the lymph node ratio (LNR), was grouped as low grade and high grade LNR, included 12 patients $(55 \%)$ and 10 patients $(45 \%)$, respectively as shown in Table 1, LNR was found to have a significant statistical correlation with lymphovascular invasion $(\mathrm{p}<0.05)$ and perineural invasion $(\mathrm{p}<0.05)$.

Statistically, significant correlation was also seen between LNR and TNM stage $(p<0.05)$, the frequency of high grade LNR was seen to increase with advancing stage as depicted in Figure 1. It was also noted that $50 \%$ of moderately differentiated and $67 \%$ of poorly differentiated tumors were of high grade LNR group (Table 1).

Table-1: Clinico-pathological variables v/s Lymph node ratio

\begin{tabular}{|l|l|l|l|}
\hline Clinical and Histopathological variables & $\mathbf{> 0 . 2 5}$ & $<\mathbf{0 . 2 5}$ & p-value \\
\hline Age & $7(32 \%)$ & $8(36 \%)$ & 0.8 \\
$<60(68 \%)$ & $3(13 \%)$ & $4(19 \%)$ & \\
$>60(32 \%)$ & & & \\
\hline Sex & $6(27 \%)$ & $9(41 \%)$ & 0.5 \\
Male (68\%) & $4(19 \%)$ & $3(13 \%)$ & \\
Female (32\%) & & & \\
\hline Tumor size & $6(27 \%)$ & $6(27 \%)$ & 0.5 \\
$<6$ cm (54\%) & $4(19 \%)$ & $6(27 \%)$ & \\
$>6 c m(46 \%)$ & $6(27 \%)$ & $6(27 \%)$ & \\
\hline Tumor type & $6(27 \%)$ & $4(18 \%)$ & 1 \\
Adenocarcinoma- Intestinal type (55\%) & $3(14 \%)$ & $2(9 \%)$ & 0.4 \\
Adenocarcinoma- Diffusely infiltrating (45\%) & $7(32 \%)$ & $10(45 \%)$ & \\
\hline Bormann type & $2(9 \%)$ & $1(4 \%)$ & 0.3 \\
III (23\%) & $5(23 \%)$ & $5(23 \%)$ & \\
IV (77\%) & $3(14 \%)$ & $6(27 \%)$ & \\
\hline Tumor differentiation & & & \\
Well differentiated (13\%) & $1(4 \%)$ & $11(50 \%)$ & \\
Moderately differentiated (46\%) & $9(42 \%)$ & $1(4 \%)$ & 0 \\
Poorly differentiated (41\%) & $9(42 \%)$ & $2(8 \%)$ & 0.01 \\
\hline Stage & $1(4 \%)$ & $10(46 \%)$ & \\
II (54\%) & $7(32 \%)$ & $3(13 \%)$ & 0.04 \\
III (46\%) & $3(13 \%)$ & $9(42 \%)$ & \\
\hline Lymphovascular invasion & & \\
Present (50\%) & & \\
Absent (50\%) & & \\
\hline Perineural invasion & & \\
Present (45\%) & & \\
Absent (55\%) & & \\
\hline
\end{tabular}

$\mathrm{p}=<0.05$ is considered significant

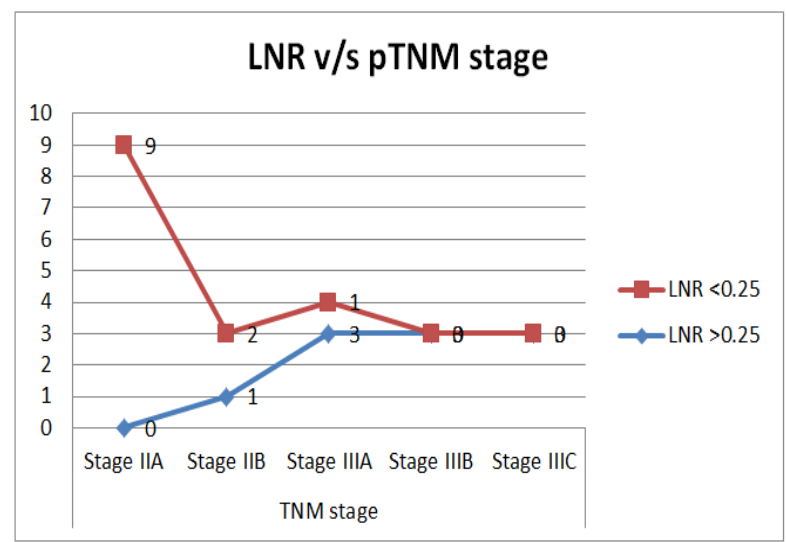

Fig-1: Association of LNR with pTNM stage of gastric carcinoma $(\mathbf{p}=\mathbf{0 . 0 3})$
The median of total number of lymph nodes isolated was 12 , ranging from $0-26$, and the number of involved lymph nodes ranged from $0-21$. In the current study according to the number of positive lymph nodes the cases were categorized as the one's with $<4$ and $>4$ positive lymph nodes respectively. We found $37 \%$ cases with $>4$ metastatic lymph nodes.

There was no significant association between LNR and hyperplastic areas (7cases), intestinal metasplasia (13cases) in adjacent stomach and dysplasia (5cases) in adjacent stomach. 
Size of largest metastatic tumor deposit and extracapsular extension in the node positive tumors was also checked. However, no association was found with LNR.

\section{The relation between Lymph node ratio and lymph nodes retrieved}

The number of metastatic lymph nodes was found to be significantly related to the total number of lymph nodes dissected $(\mathrm{r}=0.48, \mathrm{p}=<0.05)$. This is represented in Figure 2.

The total number of lymph nodes isolated did not show any significant relation with the $\operatorname{LNR}(\mathrm{r}=0.12$, $\mathrm{p}=0.6$ ); however, it was significantly associated with the number of positive lymph nodes $(\mathrm{r}=0.8, \mathrm{p}<0.001$, table2). This implies that LNR is directly related only to the nodal metastatic status irrespective of the total number of lymph nodes isolated.

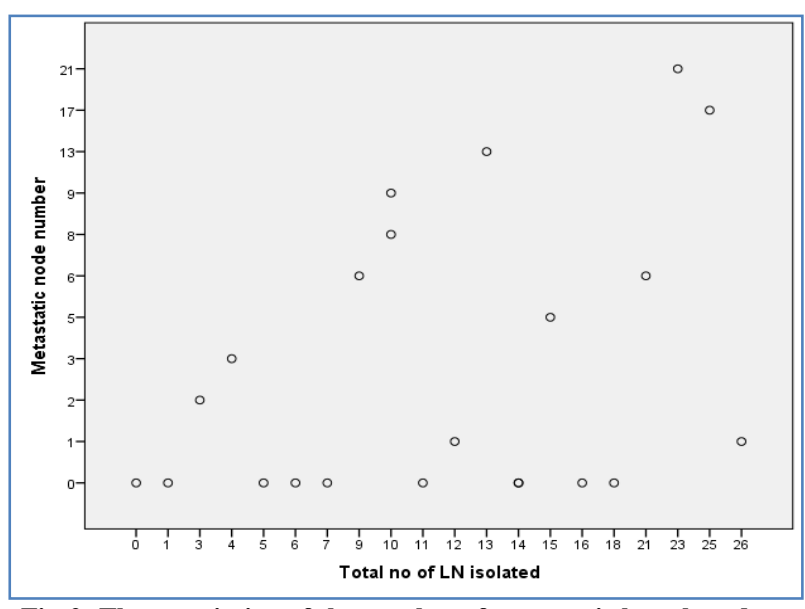

Fig-2: The association of the number of metastatic lymph nodes and the total number of lymph nodes isolated.

Table-2: No of positive lymph nodes v/s LNR

\begin{tabular}{|l|l|l|l|l|l|}
\hline \multicolumn{2}{|c|}{} & \multicolumn{2}{|l|}{ LNR } & \multirow{2}{*}{ Total } & \multirow{2}{*}{ p-value } \\
\cline { 3 - 5 } & $>\mathbf{0 . 2 5}$ & $<\mathbf{0 . 2 5}$ & & \\
\cline { 1 - 5 } No of positive lymph nodes & $>4$ & $8(36 \%)$ & 0 & $8(36 \%)$ & \multirow{2}{*}{0.00} \\
\cline { 2 - 5 } & $<4$ & $2(9 \%)$ & $12(55 \%)$ & $14(64 \%)$ & \\
\hline Total & 10 & 12 & 22 & \\
\hline
\end{tabular}

The median follow up duration was 12.5 (range 1-43) months. Twelve of these patients (54\%) received post-operative adjuvant chemotherapy with 5FU. Seventeen $(77 \%)$ of these patients died during this follow up period, one of them succumbed to recurrence with liver metastases following chemotherapy.

However, the significance in the overall survival rate in the two groups of LNR could not be ascertained due to a low sample size.

\section{DISCUSSION}

Gastric cancer is reported to be one of the most common malignancies, being the third leading cause of cancer deaths around the world. Hence extensive research and studies have been conducted to determine the factors that affect the prognosis after curative surgery. Lymph node metastasis is undoubtedly a foremost significant prognostic factor. The AJCC TNM stage requires a minimum of 16 nodes to be isolated for accurately assessing the prognosis, which oftentimes may not be possible. Several previous studies have demonstrated that with increase in the number of positive lymph nodes, prognosis gradually decreases [3]. Various studies have also reported that the total number of lymph nodes retrieved is an important prognostic factor [3]. LNR has been considered to have an even better prognostic value, higher than the TNM stage system and also to reduce stage migration [11, 12].

Here, in this study we have tried to evaluate the prognostic significance of LNR, which could be used as a new variable to assess the prognosis after curative surgery in gastric carcinoma.

We categorised the cases based on the LNR group as high grade and low grade lymph node groups with LNR $>0.25$ and $<0.25$ respectively as done by $\mathrm{Hu}$ et al. [4]. We adopted this cut-off value for LNR owing to the convenience, ease and also smaller sample size in our study.

Our study found a significant correlation between LNR and lymphovascular invasion and perineural invasion. Deng et al. and Li et al. have in their study concluded that LVI and PNI are independent prognostic factors in gastric carcinoma $[9,10]$. In the current study, LVI $(\mathrm{p}<0.05)$ and PNI $(\mathrm{p}<0.05)$ were found to have a significant statistical correlation with LNR.

LNR also showed a positive correlation with the advancing TNM stage of tumor. This finding is supported by studies done by $\mathrm{Hu}$ et al. [4].

Study conducted by Fukuda et al. reported that metastatic lymph node ratio was a very significant prognostic element in patients with gastric carcinoma in comparison with the UICC/AJCC and JGCA (Japanese Gastric Cancer Association) systems. Fukuda et al. also reported that the number of gastric lymph nodes show individual variability and the total number of lymph nodes harvested also differs. But the number of positive lymph nodes is dependent on total no of lymph nodes harvested which is in turn dependant on the surgeon and the precision of pathological evaluation [11]. It was also stated in the same study that the patients with gastric 
cancer having the same grade as per the MLR-based system are found to have the same clinical outcomes regardless of the number of lymph nodes dissected [11].

In this study we categorized cases based on the number of positive lymph nodes as cases with $<4$ and cases with $>4$ positive lymph nodes. This was based on studies done by Ichikura et al. and Gunji et al. reported that the patients with $<4$ metastatic lymph nodes had a significantly longer survival rate compared to that of patients with 4 or more metastatic lymph nodes [13-15].

The LNR in our study, showed a significant relationship with the number of positive lymph nodes but not with the total number of lymph nodes retrieved. This is similar to the studies reported by Ke et al. [16]. This indicates that the patients of gastric carcinoma with a higher LNR would have a higher risk of death post curative surgery and a shorter survival period as reported by $\mathrm{Ke}$ et al. Their results also showed that LNR could be a useful tool to predict survival for stage III gastric carcinoma patients, regardless of the total number of lymph nodes dissected [16].

Studies done by $\mathrm{Xu}$ et al. also found higher lymph node ratio to be an independent prognostic factor [17]. Lee et al. also concluded that metastatic LNR could provide a better survival information than that of the AJCC pN stage[18]. Bouliaris et al. conducted a retrospective study which incuded 110 patients. They concluded that though lymph node status and lymph node ratio had a comparable prognostic significance, lymph node ratio was found to be independent of the number of lymph nodes isolated [7].

An accurate assessment of the correlation of lymph node ratio and survival period could not be assessed in this study due to a low sample size.

\section{CONCLUSION}

Metastatic lymph node ratio could be considered as a valuable prognostic factor and could be used as an additional tool with the TNM staging system to assess the prognosis in patients after surgery. But this does not necessarily mean that the retrieval of lymph nodes could be neglected. However in certain cases where the dissection of optimum number of lymph nodes may not be possible, LNR could be used as a prognostic tool. Nonetheless, this study does have the limitations of it being a retrospective one and a smaller sample size. Also the optimum cut-off value for LNR is not yet standardised. Hence a study with larger sample size may be required to ascertain our findings and to determine the optimum cut-off value. In future LNR could be included as an additional category in the TNM staging system.

\section{REFERENCES}

1. Gco.iarc.fr. $2018 \quad$ Available from:
http://gco.iarc.fr/today/data/factsheets/cancers/7-
Stomach-fact-sheet.pdf [Accessed 4th August 2019].

2. Murugesan, C. S., Manickavasagam, K., Chandramohan, A., Jebaraj, A., Jameel, A. R. A., Jain, M. S., \& Venkataraman, J. (2018). Gastric cancer in India: epidemiology and standard of treatment. Updates in surgery, 70(2), 233-239.

3. Hou, Y., Wang, X., \& Chen, J. (2018). Prognostic significance of metastatic lymph node ratio: the lymph node ratio could be a prognostic indicator for patients with gastric cancer. World journal of surgical oncology, 16(1), 198.

4. Hu, M., Zhang, S., Yang, X., Shen, Y., Li, Z., Zhao, Y., ... \& Wang, Y. (2018). The prognostic value of lymph node ratio for local advanced gastric cancer patients with adjuvant chemoradiotherapy after D2 gastrectomy. Medicine, 97(44).

5. Amin, M. (2017). AJCC cancer staging manual. 8th ed. New York: Springer, 203-17.

6. Liu, C., Lu, P., Lu, Y., Xu, H., Wang, S., \& Chen, J. (2007). Clinical implications of metastatic lymph node ratio in gastric cancer. BMC cancer, $7(1)$, 200.

7. Bouliaris, K., Rachiotis, G., Diamantis, A., Christodoulidis, G., Polychronopoulou, E., \& Tepetes, K. (2017). Lymph node ratio as a prognostic factor in gastric cancer patients following D1 resection. Comparison with the current TNM staging system. European Journal of Surgical Oncology (EJSO), 43(7), 1350-1356.

8. $\mathrm{Xu}, \mathrm{D} . \mathrm{Z}$. (2010). The lymph node ratio staging system in gastric cancer. Annals of surgical oncology, 17(2), 659-659.

9. Deng, J., You, Q., Gao, Y., Yu, Q., Zhao, P., Zheng, Y., \& Teng, L. (2014). Prognostic value of perineural invasion in gastric cancer: a systematic review and meta-analysis. PloS one, 9(2), e88907.

10. Li, P., He, H. Q., Zhu, C. M., Ling, Y. H., Hu, W. M., Zhang, X. K., ... \& Cai, M. Y. (2015). The prognostic significance of lymphovascular invasion in patients with resectable gastric cancer: a large retrospective study from Southern China. BMC cancer, 15(1), 370.

11. Fukuda, N., Sugiyama, Y., Midorikawa, A., \& Mushiake, H. (2009). Prognostic significance of the metastatic lymph node ratio in gastric cancer patients. World journal of surgery, 33(11), 23782382.

12. Bando, E., Yonemura, Y., Taniguchi, K., Fushida, S., Fujimura, T., \& Miwa, K. (2002). Outcome of ratio of lymph node metastasis in gastric carcinoma. Ann Surg Oncol. 9(8), 775-784.

13. Deng, J. Y., \& Liang, H. (2014). Clinical significance of lymph node metastasis in gastric cancer. World journal of gastroenterology: WJG, 20(14), 3967.

14. Ichikura, T., Tomimatsu, S., Okusa, Y., Uefuji, K., \& Tamakuma, S. (1993). Comparison of the prognostic significance between the number of 
metastatic lymph nodes and nodal stage based on their location in patients with gastric cancer. Journal of clinical oncology, 11(10), 18941900.

15. Gunji, Y., Suzuki, T., Hori, S., Hayashi, H., Matsubara, H., Shimada, H., \& Ochiai, T. (2003). Prognostic significance of the number of metastatic lymph nodes in early gastric cancer. Digestive surgery, 20(2), 148-153.

16. Ke, B., Song, X. N., Liu, N., Zhang, R. P., Wang, C. L., \& Liang, H. (2014). Prognostic value of the lymph node ratio in stage III gastric cancer patients undergoing radical resection. PloS one,9(5), e96455.
17. Xu, D. Z., Geng, Q. R., Long, Z. J., Zhan, Y. Q., Li, W., Zhou, Z. W., ... \& Liu, Q. (2009). Positive lymph node ratio is an independent prognostic factor in gastric cancer after $\mathrm{d} 2$ resection regardless of the examined number of lymph nodes. Annals of surgical oncology, 16(2), 319-326.

18. Lee, S. Y., Hwang, I., Park, Y. S., Gardner, J., \& Ro, J. Y. (2010). Metastatic lymph node ratio in advanced gastric carcinoma: a better prognostic factor than number of metastatic lymph nodes?. International journal of oncology, 36(6), 1461-1467. 